\title{
Hepatic abscess
}

\author{
Miguel Quirch MD, Hawa Edriss MD
}

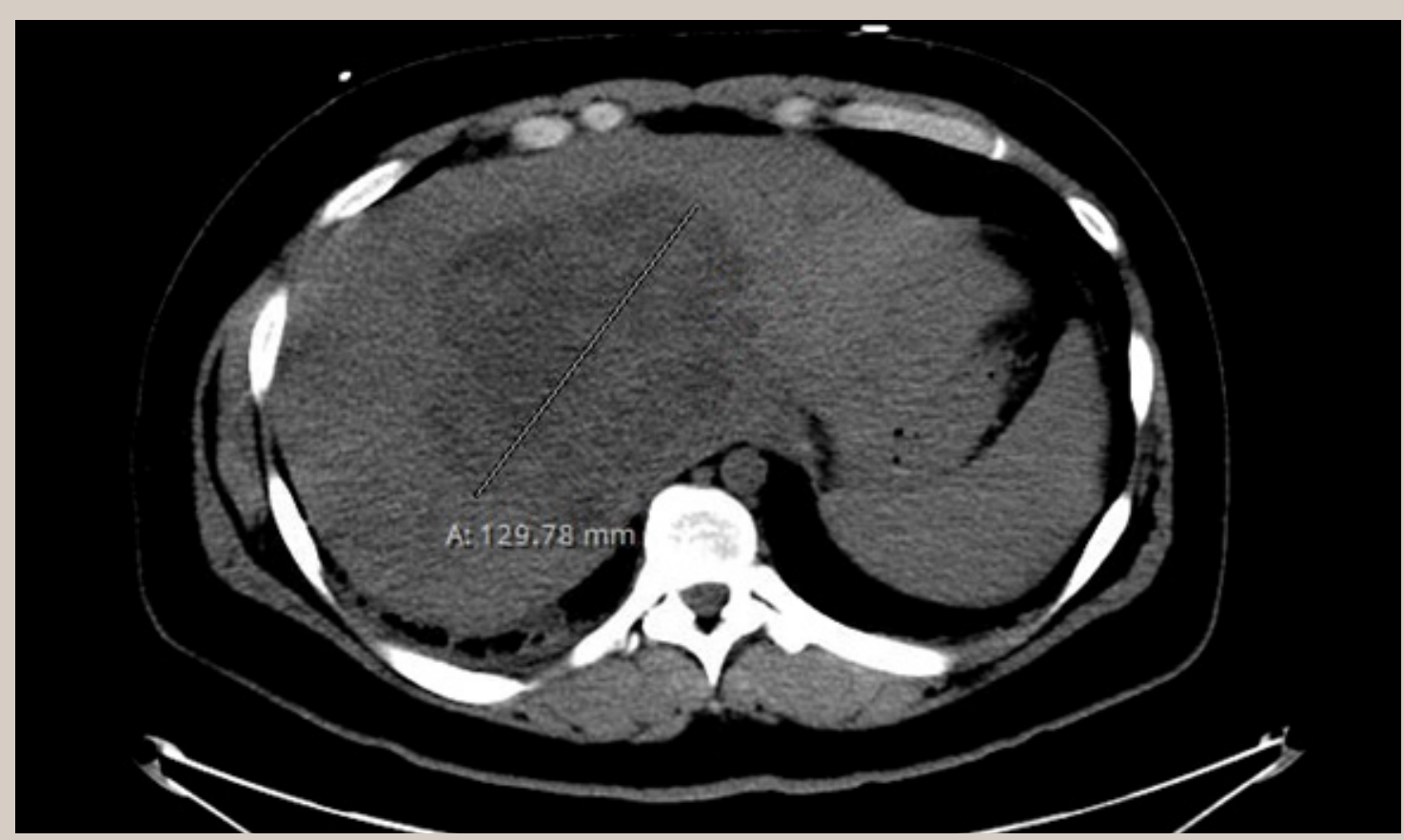

Figure 1. Initial CT scan obtained in the emergency center shows a large mass in both the right and left hepatic lobes $>10 \mathrm{~cm}$ in diameter. It has complex densities with multiple thick septae running throughout. The mass appears heterogeneous, and it is difficult to determine its exact density.

A 29-year-old Hispanic man from prison with no significant past medical history presented with excessive thirst, vomiting, and diarrhea for one week and subjective fever and chills for one month. The patient denied abdominal pain. On arrival, his temperature was $99^{\circ} \mathrm{F}$, heart rate 108 beats/minute, respiratory rate 36 breaths/minute, and SpO2 94\%. His physical examination was significant for dry mucous membrane and icteric sclera. Laboratory studies revealed a white blood count of $34.9 \mathrm{k} / \mu \mathrm{L}$, INR 1.6 , amylase 169 unit/L, lipase 1060 unit/L, BUN 94 mg/

Corresponding author: Miguel Quirch Contact Information: Miguel.quirch@ttuhsc.edu DOI: 10.12746/swrccc 2016.0416.222 $\mathrm{dl}$, creatinine $6.4 \mathrm{mg} / \mathrm{dL}$, sodium $120 \mathrm{mmol} / \mathrm{L}$, bicarbonate $16 \mathrm{mmol} / \mathrm{L}$, alkaline phosphate $275 \mathrm{unit} / \mathrm{L}$, total bilirubin $8.2 \mathrm{mg} / \mathrm{dl}$, direct bilirubin of $6.9 \mathrm{mg} / \mathrm{dl}$, and AST/ALT of $73 / 57$ unit/L. Computed tomography of the abdomen revealed a hepatic mass greater than $10 \mathrm{~cm}$ in diameter with multiple thick septae in both the right and left hepatic lobes suspicious for abscess or tumor (Figure). Amoeba and echinococcus ELISA tests and an E. histolytica antigen assay were negative. The patient underwent CT guided drainage by interventional radiology (IR). Blood and hepatic drainage cultures grew Streptococcus intermedius; the aspirate had a negative AFB stain. The patient was initially treated with broad-spectrum antibiotics (IV meropenem and vancomycin) and then switched to IV ceftriaxone based on sensitivity results. He subse- 
quently underwent two more IR drainage procedures with a catheter left in place. Since patient continued to have bloody drainage, the surgery team was consulted, and they recommended a liver biopsy to exclude malignancy. A surgical biopsy showed reactive liver tissue with cholestasis and no malignancy.

A pyogenic liver abscess usually presents with nonspecific symptoms and can take from two weeks to more than one month to develop and present with symptoms. These symptoms typically include malaise, fever/chills, nausea/vomiting, weight loss; only half of patients have right upper quadrant symptoms. Poor prognostic indicators include a bilirubin $>3.5 \mathrm{mg} /$ $\mathrm{dl}$ and the presence of multiple abscesses. Mortality ranges between 3-30\% even with early identification and optimal therapy. The three most common etiological causes of liver abscess are polymicrobial (pyogenic) infection which accounts for $80 \%$ of cases, amebic infection (10\%), and fungal infection (<10\%), most frequently due to Candida species. After right upper abdominal quadrant pathology is suspected, an abdominal ultrasound is usually the first imaging study ordered. Ultrasonography uses high frequency sounds that are deflected, refracted, and reflected off tissue to create an image, with the boundaries between tissue forming visible differences on the images. Ultrasound can help to distinguish biliary disease from hepatic disease; it identifies pathology $>1 \mathrm{~cm}$ in diameter. ${ }^{1}$ It is particularly useful in identifying amebic abscesses which typically appear as oval or round masses near the liver capsule, are hypoechoic with low-level internal echoes, and do not have significant wall echoes compared to pyogenic abscesses, which can appear as either discrete hypoechoic nodules or as ill-defined areas of distortion. ${ }^{2}$ Abdominal CT scan is the next step and provides information on the extent of disease and precise localization for guided drainage. Computed tomography uses an emitter sending x-ray beams through tissue to a detector, forming cross sections of tissue. A hepatic abscess appears as a well-demarcated mass, hypodense to surrounding liver parenchyma. A double target sign on dynamic studies and an enhanced rim sign can be seen when contrast used. Gas is detected in $20 \%$ of these lesions. It is more sensitive than ultrasound in detecting pyogenic abscess (95-100\% vs $80-90 \%)$, solid masses, and small microabscesses, but it is not as accurate distinguishing amebic abscesses when compared to ultrasound. Radiography of the chest helps exclude pleural or pulmonary pathologies and can identify extension or rupture of an abscess. Therapeutic percutaneous needle aspiration (PNA) or percutaneous catheter drainage (PCD) and antibiotics are needed for definitive treatment. Surgical exploration is reserved for large abscesses $>5 \mathrm{~cm}$ in size, complicated abscesses, and abscesses resistant to treatment. ${ }^{1}$ Older studies suggested that serial PNAs were the best treatment option, but more recent studies have shown that PCD has higher success rates, reduces the time required to achieve clinical relief, and results in a $50 \%$ reduction in abscess cavity size. ${ }^{3}$ Provided that the abscess resolves, both drainage methods result in comparable long term outcomes. ${ }^{4}$

\footnotetext{
Author Affiliation: Miguel Quirch is a resident in Internal Medicine at Texas Tech University Health Sciences Center in Lubbock, TX. Hawa Edriss is a fellow in Pulmonary and Critical Care Medicine at TTUHSC in Lubbock, TX.

Reviewer: Eman Attaya MD

Received: 8/7/2016

Accepted: 9/19/2016

Published electronically: 10/15/2016
}

\section{References}

1. Vogt D, Ferri F, Anand B. Liver Abscess. ClinicalKey, Elsevier, BV, February 2, 2012. (Accessed August 25, 2016, at https://www-clinicalkey-com/\#!/content/medical_topic/21-s2.0-1010020).

2. Mortelé K, Segatto E, Ros P. The infected liver: radiologic-pathologic correlation. RadioGraphics 2004; 24(4):937-955. 3. Yu S, Ho S, Lau W et al. Treatment of pyogenic liver abscess: Prospective randomized comparison of catheter drainage and needle aspiration. Hepatology 2004 39(4):932-938.

4. Cai Y, Xiong X, Lu J et al. Percutaneous needle aspiration versus catheter drainage in the management of liver abscess: a systematic review and meta-analysis. International Hepato-Pancreato-Biliary Association 2015; 17(3):195-201. 